\title{
Un nouvel espace scientifique pour la francophonie médicale
}

Chacun, parmi nous, a l'intuition de la nécessité de l'espace culturel francophone. Chacun ressent que nous ne pouvons demeurer dans un monde standardisé, uniculturel, dont l'uniformité fait craindre soumission et frustration. Chacun aussi craint un monde d'affrontements et de combats idéologiques. $L$ a francophonie, dans ce contexte, offre une alternative culturelle et comportementale : à l'affrontement, elle préfere l'ouverture, et elle propose le débat comme substitut au combat.

Encore faut-il que la francophonie se donne les moyens de son efficacité. Q uand on parle de moyens, on pense immédiatement aux aspects financiers, au soutien matériel des actions.

Les efforts supplémentaires de la France vers I'A gence U niversi taire Francophone permettent à sa rectrice $M$ adame $G$ endreau$M$ assaloux d'envisager des obj ectifs encore plus ambitieux dans tous les secteurs universitaires et de ne pas craindre l'avenir. $M$ ais les moyens ne peuvent pas toujours venir de l'extérieur de nos institutions, et ils ne consistent pas uniquement en subsides supplémentaires. Les moyens viennent de nous mêmes, spécialement de notre capacité d'imagi nation et de notre propension à nous unir.

La Soci été Internationale $F$ rancophone d'Education M édicale (SIFEM) témoigne de cette ambition de la francophonie. $N$ é à I'occasion des J ournées de P édagogie de la CIDMEF en avril dernier, cette jeune société couronne des années d'efforts intellectuels des en seignants en médecine, qui souvrent en même temps à leurs collègues des autres filières de santé. La tâche de la SIFEM est triple: consacrer la sci ence pédagogique en installant un espace de débat chez les enseignants en santé, promouvoir la recherche dans cette disci pline, être un fer de lance de la francophonie.

\section{C onsacrer la pédagogie comme une science des professions de santé}

Le défi n'est pas mince. $D$ ans des métiers comme les nôtres, où la pratique médicale l'emporte souvent sur les objectifs de formation, où le compagnonnage est vécu comme la seule méthode educati onnelle qui vaille, I'investi ssement en pédagogi e, ressenti d'abord comme inutile, surprend puis séduit. M ais trop rares sont les Facultes où la pédagogie est présente de façon institutionnelle: il y a loin d'exister partout une cellule de pédagogie, ou un enseignement de la façon d'enseigner. Trop rares, aussi, sont les disciplines qui créent en leur sein un noyau de pédagogues pour promouvoir et rendre plus effici ent leur en sei gnement. $\mathrm{N}$ otre soci été doit donc rendre visibles les efforts de ceux qui ont été les pionniers; en les groupant, elle les fédère, les rend plus cré dibles et plus pui ssants. D otée de notre revue "Pédagogie M édicale », la SIFEM devient donc la soci été sci entifique que l'espace francophone attendait.

La fondation de notre soci été est par ailleurs concomitante de trois phénomènes qui justifient encore plus son existence : la transmissi on informatique des savoirs, les nécessi tés de la formation continue des médecins, le rapprochement en éducation des différents professi onnels de santé. O n sent partout, même si parfois on la craint, la nécessité d'une banque francophone des connai ssances médicales.

Des initiatives ont déjà été prises, la première étant celle de la CID M EF sousl'autorité de Jean-François $D$ enef, puis Christian Binet; à partir des universi tes françai ses, se développe en parallèle l'U niversité M édicale 
Virtuelle Francophone dont les efforts devraient rejoindre ceux de la CIDM EF.

On voit bien le rôle que devrait jouer la SIFEM tant dans le développement de cette entreprise, que dans celui de l'organisation des actions, et la validation des méthodes. La formation médicale continue se cherche elle aussi ; parfois obligatoi re, toujours nécessaire, elle nécessite une réfl exion permanente sur ses méthodes et son évaluation.

Enfin, le rapprochement des préoccupations en formation des professions qui nous sont proches nous obli ge à nous pencher ensemble sur la pédagogie, dans des domaines qui nous sont communs, mais où les objectifs sont différents: là encore notre réflexion ne peut qu'être facilitée par la SIFEM.

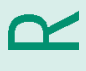

\section{Promouvoir la recherche en pédagogie}

D evenir la soci été savante de la pédagogie implique de dynamiser la recherche de cette discipline. II faut pour cela que la SIFEM recherche des «succursales » dans chacune de nos facultés et chacune des disciplines médicales. II faut aussi qu'elle comprenne l'étendue du terrain pédagogique.

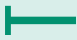

N otre société ne jouera réellement son rôle que si elle favorise la création, dans chacune de nos institutions, d'une "maison de la pédagogie », c'est-à-dire d'au moins une cellule dirigée par un expert en pédagogie, telle que l'a défini la CIDM EF (degré 3 ) et des enseignants les plus impliqués, avec un secrétariat mi nimum. C'est à partir de cette cellule que pourront se développer des travaux, des thèses, la formation des jeunes en sei gnants. Chacune des sociétés savantes, qu'elleappartienne aux sci ences cliniques ou biologiques, devrait bénéficier d'une structure de réflexion analogue.

Enfin, la SIFEM doit favoriser tous les aspects typi quement médicaux de son champ de compétence. II n'est pas suffisant de se pencher sur les méthodes de transmi ssion des connaissances, sur la docimologie. II faut aussi aller vers l'éducation du regard pour les spéciali stes de l'i mage, sur l'éducation du geste pour tous ceux qui doivent acquérir des excellences manuelles à travers la mise au point de simulateurs, etc.

\section{Être un fer de lance de la francophonie}

La SIFEM doit être une société ambitieuse et élitiste. C'est en effet, et en premier lieu, un espace de réflexion qui réunit tous les enseignants en médecine de la francophonie pour améliorer la formation des médecins. C'est aussi l'espace de création des nouveaux concepts de cette jeune sci ence.

Tirant les ensei gnants vers le haut, elle doit aussi les unir. Elle peut devenir la fédération de toutes les cellules de pédagogie des facultés. En union avec la CIDMEF, elle peut créer des journées permettant la publication de communications de haute tenue. Comme nous le disions plus haut, elle doit jouer un rôle primordial dans le développement des espaces virtuels de formation.

Elle servira ainsi d'exemple pour les autres champs de la francophonie qui ne pourront que sen inspirer. A l'instar de la CID MEF, elle deviendra un des plus beaux fleurons de I'A gence U niversitaire de la Francophonie.

La SIFEM, lieu de rencontre de tous ceux qui font progresser la pédagogie, offre ainsi à la francophonie un nouvel espace scientifique et lui trace un nouveau défi.

Jacques Roland Doyen honoraire de la Faculté de M édecine de N ancy 